\title{
Ag-Au alloy nanoparticles: synthesis and in situ monitoring SERS of plasmonic catalysis
}

\author{
Qingyan Han, Chengyun Zhang, Wei Gao, Zhihang Han, Tingzhuo Liu, Caixia Li, \\ Zhaojin Wang, Enjie He, Hairong Zheng* \\ School of Physics and Information Technology, Shaanxi Normal University, 710119, \\ Xi'an, China \\ *Corresponding author: E-mail: hrzheng@ snnu.edu.cn.
}

\begin{abstract}
Noble metal nanostructures are currently of great interest for their unique plasmonic property and potential applications in catalysis and surface-enhanced spectroscopy. However, the application of plasmonic nanostructures for quantitatively in situ SERS monitoring of the catalytic reaction has been a great challenge for investigators because combining plasmonics with catalysis requires the same kind of noble metal nanoparticles (NPs) in two very different size regimes. Herein, We have demonstrated a facile wet chemical method to synthesize Au-Ag alloy plasmonic NPs that could combine the desired plasmonic and catalytic properties with same NPs. The catalytic activity of Au-Ag alloy NPs using the reduction of 4-nitrothiophenol (4-NTP) by sodium borohydride $\left(\mathrm{NaBH}_{4}\right)$ is chosen as a model reaction. The signals of the reaction processes are detected and identified through in situ SERS spectroscopy with high sensitivity. The insights gained by current study may serve as a promising and powerful technique for better investigation in the heterogeneous catalysis. Moreover, the reduction of aromatic nitro compounds with prepared Au-Ag alloy NPs also provides potential application in sewage treatment.
\end{abstract}

Keywords: Au-Ag alloy nanoparticles, surface plasmon resonance, surface-enhanced Raman scattering, plasmonic catalysis

\section{Introduction}

Plasmonic metal nanocrystals exhibit unique properties with potential application 
in many fields, which include catalysis [1], photovoltaics [2], sensing [3], and photothermal therapy [4]. The plasmonic responses strongly depend on the type, size, configuration and the surrounding environment of the noble metal nanocrystals [5-7]. Recently, through integrating two or more metal materials into a single entity, the plasmonic property can be significantly extended due to synergistic effect $[1,8]$. For example, hybrid, core-shell or alloy structures can produce superior and sometimes unprecedented physical and chemical properties in catalysis and optics for both fundamental investigations and technological applications [9, 10]. Various metal nanocrystals have been prepared using wet chemistry methods $[6,11]$, which produce plasmonic resonances in the visible to near-infrared regions, facile surface conjugation to a variety of chemical and biomolecular ligands [12], biocompatibility and catalytic effect towards many important oxidization reactions [8, 13]. These applications in catalysis could improve the feasibility of wastewater treatment technique.

To investigate metal-catalyzed reactions, several kinds of technique have been successfully employed for conducting the necessary measurement, which include UV-vis-infrared absorption [14, 15], Raman or surface-enhanced Raman scattering (SERS) spectroscopy $[16,18]$. However, the absorption in UV-vis regime can only offer very limited chemical information and the application of infrared spectroscopy to aqueous system is not a good choice due to strong water absorption of the system. Compared with optical absorption, Raman scattering, especially SERS has obvious advantages for in situ monitoring the interfacial reaction. SERS mainly utilizes the enhanced electromagnetic field and localized surface plasmon resonance (LSPR) to get enhanced Raman scattering signal from target molecules, which is generated at some metal surfaces upon laser excitation $[19,20]$. Additionally, the technique of SERS spectroscopy can provide non-destructive and ultra-sensitive characterization down to a single-molecule regime [21]. Thus, as a powerful analysis and characterization technique, SERS-based signal detection and molecular identification combine the advantages of high chemical specificity (vibrational Raman scattering), 
high sensitivity (electromagnetic and chemical enhancement), and surface selectivity (near-field enhancement) [22-24]. Unfortunately, the combination of desired plasmonic and superior catalytic properties on the same kind of noble metal nanocrystals has been a challenge because they typically require metal nanoparticles (NPs) in two drastically different size regimes [25]. For example, small Au NPs have shown good catalytic activity, they are active even in at room temperature [26, 27], but the efficient Raman or SERS signals are difficult to be detected because of their very small scattering cross sections. On the other hand, larger Au NPs show geometry-dependent optical properties that can provide necessary SERS enhancement [17], but their catalytic activity decay rapidly. Ag NPs have much stronger surface plasmon resonance than $\mathrm{Au}$ due to its proper electronic structure and dielectric function [12], but they have not been fully used in practical applications due to their poor chemical stability and aggregation effect [28]. Therefore, it is very important to combine superior catalytic and high plasmonic activity simultaneously in one nanostructure.

In current paper, a strategy to facilely synthesize Au-Ag alloy NPs with good dispersibility in aqueous solution is presented. By adjusting the ratio of two metals, we obtain a tunable localized surface plasmon resonance (LSPR) with high intensity in visible region. The Au-Ag alloy NPs show excellent SERS characteristics and high catalytic activity. Their potential application for in situ SERS monitoring of the quantitative reaction of 4- nitrothiophenol (4-NTP) reduction to 4-aminothiophenol (4-ATP) by $\mathrm{NaBH}_{4}$ in colloidal is presented too.

\section{Experimental}

\subsection{Materials}

Sodium citrate $\left(\mathrm{C}_{6} \mathrm{H}_{5} \mathrm{Na}_{3} \mathrm{O}_{7}, 99 \%\right)$, silver nitrate $\left(\mathrm{AgNO}_{3}, 99.8 \%\right)$, hydrogen tetrachloroauric acid $\left(\mathrm{HAuCl}_{4} \cdot 4 \mathrm{H}_{2} \mathrm{O}, 99 \%\right)$, sodium borohydride $\left(\mathrm{NaBH}_{4}, 98 \%\right)$ and ethanol were supplied by Sinopharm Chemical Reagent Co., Ltd. (China). 4-nitrothiophenol (4-NTP, 80\%) was purchased from Sigma-Aldrich. All chemical reagents were used as received without further purification. Deionized (DI) water was 
used throughout experiments.

\subsection{Synthesis of Au-Ag alloy nanoparticles}

$\mathrm{Au}-\mathrm{Ag}$ alloy NPs were synthesized via reduction of $\mathrm{AgNO}_{3}$ and $\mathrm{HAuCl}_{4} \cdot 4 \mathrm{H}_{2} \mathrm{O}$ with citrate. For $\mathrm{Au}-\mathrm{Ag}$ alloy NPs, $\mathrm{HAuCl}_{4} \cdot 4 \mathrm{H}_{2} \mathrm{O}(0.01 \mathrm{M}, 1 \mathrm{ml})$ and $\mathrm{AgNO}_{3}(0.01 \mathrm{M}$, $1 \mathrm{ml}$ ) were dissolved in $78 \mathrm{ml}$ of boiled deionized water firstly while stirring. Then, 8 $\mathrm{ml}$ of sodium citrate $(0.1 \mathrm{M})$ was quickly added into the solution. In this case, the mixture was kept at $100{ }^{\circ} \mathrm{C}$ for 15 min with continuous stirring. The reaction was completed by cooling it down to room temperature. The synthesized NPs were washed and centrifuged for several times before they were dispersed in water and kept in the dark at room temperature.

\subsection{In situ monitoring of the catalytic reaction with SERS}

$10 \mu \mathrm{l}$ of $10^{-3} \mathrm{M} 4$-NTP ethanol solution was added to $1 \mathrm{ml}$ colloidal suspension of Au-Ag alloy NPs and incubated at room temperature overnight, which formed a 4-NTP molecular self-assembled monolayer (SAM) on the surface of NPs. Then the $\mathrm{Au}-\mathrm{Ag}$ alloy NPs were washed for 2 times with water and resuspended in $400 \mu \mathrm{l}$ water. $100 \mu \mathrm{l}$ of NPs-catalyzed 4-NTP reduction occurred upon the addition of $200 \mu 1$ of $10^{-2}$ $\mathrm{M} \mathrm{NaBH}_{4}$ into a $0.4 \mathrm{ml}$ quartz vessel at room temperature. The kinetics of the catalyzed reactions was measured in situ with real-time monitoring SERS.

\subsection{Characterization}

The prepared nanostructures were characterized in several ways, Transmission electron microscope (TEM) images were obtained with JEOL 2100 TEM at an acceleration voltage of $200 \mathrm{kV}$. Scanning TEM (STEM) images were collected on a FEI-Tecnai G2 F20 Field-emission transmission electron microscope (FE-TEM) operating at $200 \mathrm{kV}$ accelerating voltage. UV-visible-NIR absorption spectra were measured by using a Perkin Elmer Lambda 950 spectrometer. Raman spectral measurements were conducted with a LabRam HR Evolution Raman system (Jobin-Yvon). The wavelength of the excitation light was $633 \mathrm{~nm}$. The numerical calculation on the electromagnetic field distribution was carried out with the 
application of 3D finite element method [29].

\section{Results and discussion}

\subsection{Synthetic mechanism and plasmonic properties}

As presented in the experimental section, Au-Ag alloy NPs were prepared by using wet chemistry method. Fig. 1(a) shows the TEM image of Au-Ag alloy NPs as synthesized, which indicates the uniformity of the products. The average size of $\mathrm{Au}-\mathrm{Ag}$ alloy Nps are around $33 \pm 2 \mathrm{~nm}$. To confirm the distribution of each element in the as-prepared Au-Ag alloy NPs, FE-TEM analysis were conducted. Fig. 1 (b)- 1(e) are STEM images of typical Au-Ag alloy NPs, which were used as elemental mapping analysis in later discussion. Line-scanning profiling analysis shows that both $\mathrm{Au}$ and $\mathrm{Ag}$ distributed homogeneously across the particles (Fig. 1(b)). STEM-energy dispersive X-ray (STEM-EDX) elemental mapping of $\mathrm{Au}$ and Ag were separately shown in Fig. 1(c) and 1(d), which further conformed the composition of Au-Ag alloy NPs. The full coverage of both Au and Ag indicates the alloy structure of the sample. The merged image (Fig. 1(e)) further supports the complete overlapping of two metal components.

To characterize the property of NPs, surface plasmon resonance (SPR) was investigated by UV-visible and NIR absorption spectra. Fig. 1(f) presented normalized extinction spectra of as-prepared $\mathrm{Au}, \mathrm{Ag}$, and $\mathrm{Au}-\mathrm{Ag}$ alloy NPs. Au and Ag NPs exhibit characteristic SPR peaks at about $523 \mathrm{~nm}$ and $422 \mathrm{~nm}[30,31]$. As the ratio content of $\mathrm{Au}$ to $\mathrm{Ag}$ increases in alloy $\mathrm{Au}-\mathrm{Ag}$ NPs, spectral red-shift occurs for the SPR from the side of Ag NPs towards that of the Au NPs. The SPR peak at $\lambda_{\mathrm{SPR}}=$ 472nm for $\mathrm{Au}-\mathrm{Ag}$ presented in the figure matches well with the composition alloy NPs. The corresponding photographs shown in the inset of Fig. 1(f) demonstrate a gradual color change from yellow to wine red with the content increase of Au. All of these results confirm the formation of monodispersed Au-Ag alloy NPs in current study. 


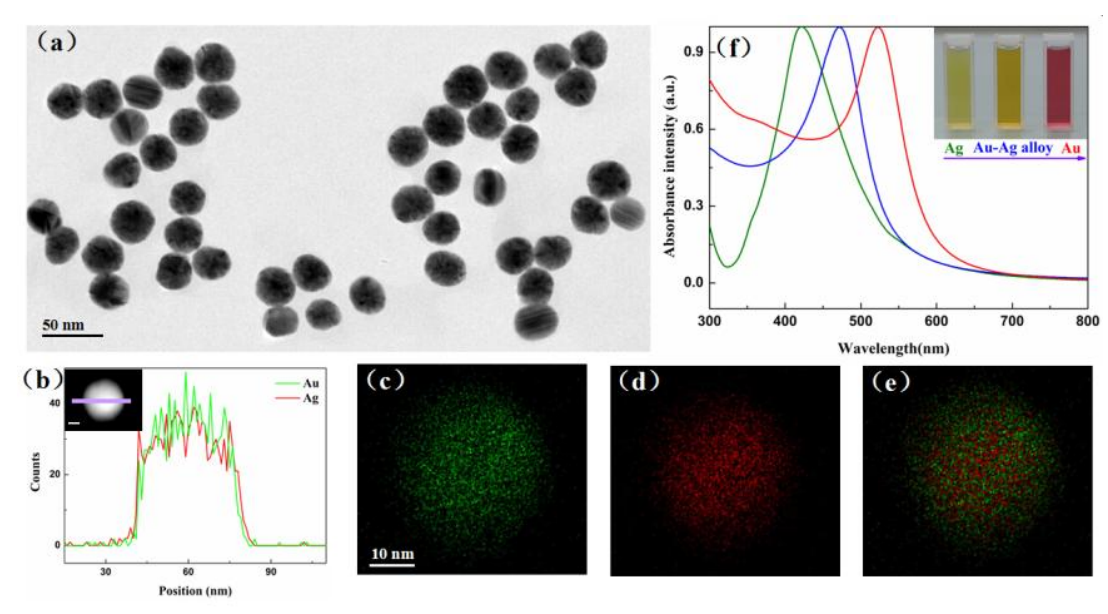

Fig. 1. Characterization of metal NPs. (a) TEM image of Au-Ag alloy NPs. (b) STEM image and cross-sectional compositional line profiles of Au-Ag alloy NPs. (c) and (d) STEM-EDX elemental mapping image of $\mathrm{Au}(\mathrm{d})$ and $\mathrm{Ag}(\mathrm{e})$ from a single $\mathrm{Au}-\mathrm{Ag}$ alloy nanoparticle. (e) The merged image of (c) and (d). (f) Absorption spectra of Ag, Au-Ag alloy and Au NPs, which correspond to green, blue and red curves. Insets are photographs of the corresponding samples.

To better understand the plasmonic property of metal NPs, the distribution of local electric field as shown in Fig. 2 was calculated by finite element method (COMSOL Multiphysics) [29]. The basic data on the shape and size of $\mathrm{Au}, \mathrm{Ag}$ and $\mathrm{Au}-\mathrm{Ag}$ alloy NPs were from the experimental measurements. Fig. 2 shows the calculated extinction spectra for an individual particle at a normally incident light excitation. The extinction peaks for Ag, Au-Ag alloy and Au NPs locate at 396, 470, and $522 \mathrm{~nm}$, respectively. Ag NPs exhibit a narrower spectral width and higher resonance intensity. The simulated electromagnetic field distributions of the Ag, Au-Ag alloy, and Au NPs under the resonance excitations are presented in the right panel of Fig. 2. It can be seen that Au-Ag alloy NPs exhibit a stronger electric field than Au NPs. Comparing with the numerical simulation, we notice that a red-shift occurred in the LSPR spectra obtained by the experimental observation, and the biggest shift presented for $\mathrm{Ag}$ particles. For the observed LSPR spectral shift, the possible assembly effect existed in the experimental measurement should be the reason of it. Since only single nanoparticle is to be considered in the calculation, therefore the corresponding experimental result for the comparison should be exactly from very well isolated 
single particles. According to the experimental investigation, the practical metal particle system usually contains more or less nanoparticle clusters during the measurement even though the characterization results present very good dispersibility. For the systems of $\mathrm{Au}, \mathrm{Ag}$ and $\mathrm{Au}-\mathrm{Ag}$ alloy NPs, Ag NPs have much lower chemical stability and are easier to be aggregated. The aggregation effect would lead to an increase of the particle size and charge separation, leading to a lower frequency for the collective oscillation of free electrons and thus a red-shift of the LSPR in the experimental observation comparing with the numerical simulation $[32,33]$.

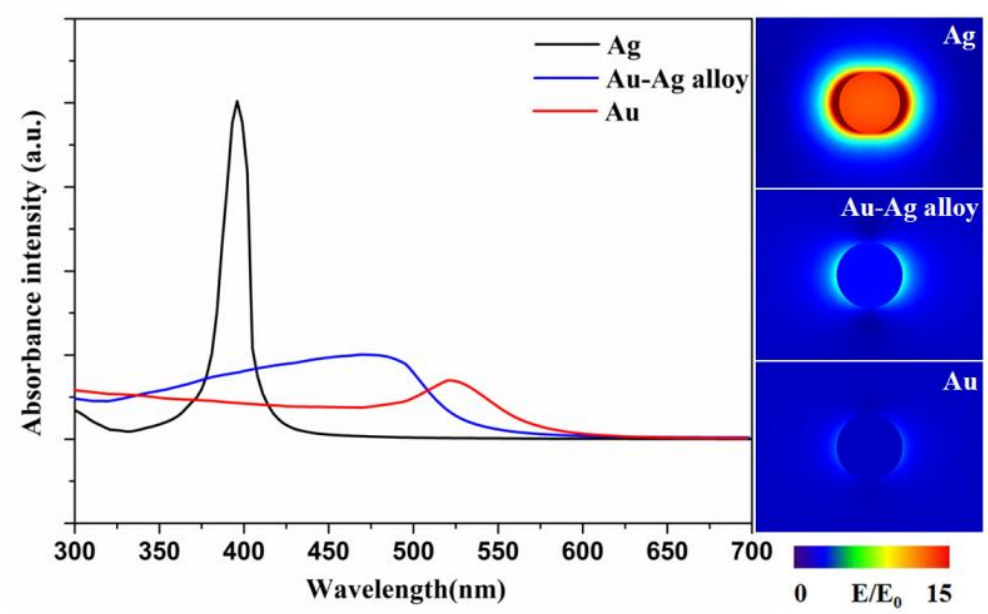

Fig. 2. Left panel: extinction spectra of $\mathrm{Ag}, \mathrm{Au}-\mathrm{Ag}$ alloy and $\mathrm{Au}$ nanoparticle in water solution, the diameter of the particle is $33 \mathrm{~nm}$. Right panel: spatial electric field distributions for $\mathrm{Ag}, \mathrm{Au}-\mathrm{Ag}$ alloy and Au nanoparticle. The color bars at the bottom represent the amplitude of $\left|\mathrm{E} / \mathrm{E}_{0}\right|$, where $\mathrm{E}$ is the local electric field around the nanoparticle, and $\mathrm{E}_{0}$ is the incident electric field.

Incident wavelength dependence of the electromagnetic field amplification is one important characters of LSPR and affect the plasmonic effect significantly. The numerical simulation the electromagnetic field distributions of the Ag, Au-Ag alloy, and Au nanoparticle are carried out with the excitation wavelength of $532 \mathrm{~nm}, 633 \mathrm{~nm}$, and 785nm (Fig. S1). The highest intensity of the electromagnetic field was obtained at the excitation of $532 \mathrm{~nm}$, while the lowest one was with $785 \mathrm{~nm}$. The intensity of the electromagnetic field obtained with $633 \mathrm{~nm}$ excitation was in between. However, when the system is excited by $532 \mathrm{~nm}$ laser, the intensities of Raman scattering bands for 4-NTP, 4-ATP, and DMAB are much weaker than that with the other wavelength 
excitation. It is reported that the intensity of the band at about $1081 \mathrm{~cm}^{-1}$ mostly depends on the enhancement caused by LSPR, and the intensity of the band at 1081 $\mathrm{cm}^{-1}$ for 4-ATP and DMAB decreases following the order of excitation wavelength of $633 \mathrm{~nm}>785 \mathrm{~nm}>532 \mathrm{~nm}$ [34]. Therefore, we took $633 \mathrm{~nm}$ laser as an excitation light in the following study.

\subsection{In situ monitoring of catalytic reaction with SERS}

Since the simultaneous introduction of $\mathrm{Ag}$ and $\mathrm{Au}$ are fulfilled, it is very important to investigate the in situ real-time monitoring SERS of catalytic reaction with presented $\mathrm{Au}-\mathrm{Ag}$ alloy NPs. Owing to the synergistic effect of the metal components, the plasmonic activity of $\mathrm{Ag}$ increases while catalytically active $\mathrm{Au}$ is presented at the same time. In contrast, no catalytic activity is observed without the introduction of $\mathrm{Ag}$. To demonstrate the catalytic and SERS activity of Au-Ag alloy NPs, a proper material for this proof-of-concept study and practical applications are required. Here, we investigate the reduction of aromatic nitro compounds $\left(\mathrm{R}-\mathrm{NO}_{2}\right)$ to the aniline derivative $\left(\mathrm{R}-\mathrm{NH}_{2}\right)$ at room temperature (Fig. 3), which would be useful for environment governance and the synthesis of fine chemical.

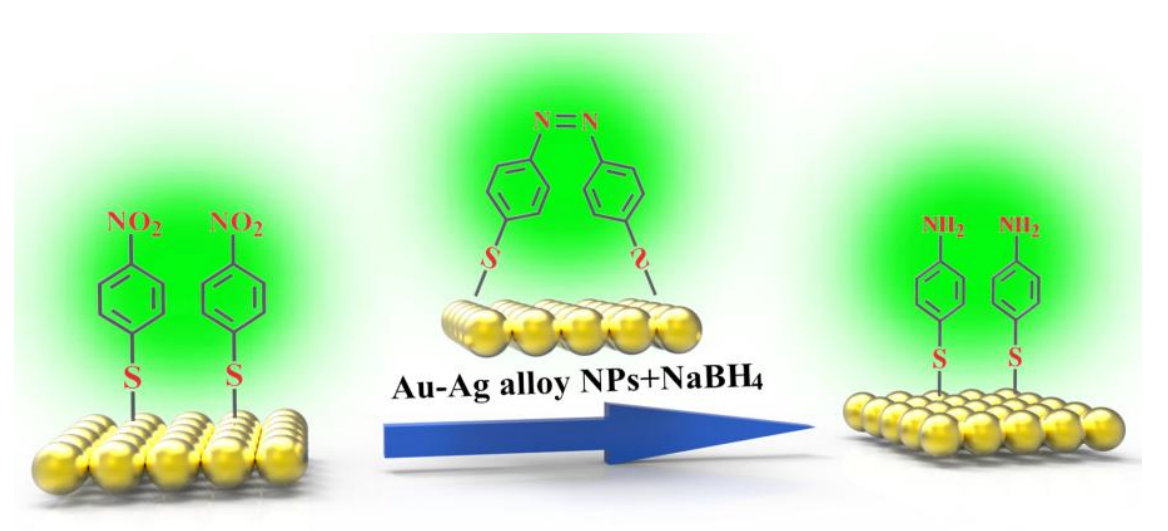

Fig. 3. A tentative mechanism of the chemical reduction of surface-adsorbed 4-NTP by $\mathrm{NaBH}_{4}$ to DMAB and finally to 4- ATP catalyzed by Au-Ag alloy NPs.

The time-resolved SERS monitoring can gain quantitative insights into the kinetics and mechanism study of nanoparticle-catalyzed reaction. Au-Ag alloy nanoparticle-catalyzed reaction through the reduction of 4-NTP by $\mathrm{NaBH}_{4}$ was chosen as a model reaction, which will be compared to the catalytic activity of $\mathrm{Ag}$ and $\mathrm{Au}$. 
Both the intrinsic reaction mechanism and kinetics can be studied in real time by online detecting SERS signals from themonolayer molecule preadsorbed on the surface of metal NPs. Thus, the identification of transient intermediates during the reaction processes became possible. To obtain the samples for conducting SERS measurement, colloidal suspension of NPs was incubated with ethanolic solution of 4-NTP at room temperature overnight, which formed a 4-NTP molecular SAM at the surface of NPs. The SAM of 4-NTP on the surface of metal NPs underwent a two-step reduction process upon the introduction of $\mathrm{NaBH}_{4}$ at room temperature, for which the final product is 4-ATP. As shown in Fig. 4, the SERS spectrum of 4-NTP monolayer exhibite four characteristic vibrational bands at $854,1081,1338$, and $1571 \mathrm{~cm}^{-1}$, which correspond to $\mathrm{C}-\mathrm{H}$ wagging, $\mathrm{C}-\mathrm{S}$ stretching, $\mathrm{O}-\mathrm{N}-\mathrm{O}$ stretching, and the phenyl ring modes, respectively [35]. However, the feature of SERS signal for 4-NTP remains unchanged at the beginning of $\sim 2$ min in the presence of $\mathrm{NaBH}_{4}$ (Fig. S2). This is most likely due to the formation of active surface hydrogen species upon the adsorption of borohydride ions onto the surface of Au-Ag NPs [36]. Therefore, the hydrogenation of 4-NTP was initiated only when the concentration of surface hydrogen species reached to the threshold. As the reaction proceeded, strong Raman signal at $1338 \mathrm{~cm}^{-1}$ decreased gradually, and new vibrational Raman signals of the intermediate at 1144, 1390, and $1436 \mathrm{~cm}^{-1}$ appeared along the reaction pathway, which were due to $\mathrm{C}-\mathrm{N}$ symmetric stretching, $\mathrm{N}=\mathrm{N}$ stretching, and $\mathrm{C}-\mathrm{H}$ in-plane bending modes of $4,4^{\prime}$-dimercapto-azobenzene (4, 4' -DMAB), respectively [37]. With increasing of reaction time (from bottom to top in Fig. 4), the intensity of SERS signal from 4, 4' -DMAB initially increases and then decreases ultimately, indicating that $\mathrm{NO}_{2}$ groups start to be reduced. The band arising from $\mathrm{C}-\mathrm{H}$ wagging and phenyl ring modes of 4-NTP at 854 and $1571 \mathrm{~cm}^{-1}$ also decreases gradually, concomitant with the intensity of a new band at $1595 \mathrm{~cm}^{-1}$ increases and an additional characteristic signal of 4-ATP at $1489 \mathrm{~cm}^{-1}$ appears (Fig. 4 top) [35]. Therefore, one can conclude that the final reaction product is 4-ATP for the reduction of 4-NTP by $\mathrm{NaBH}_{4}$, which demonstrates the catalytic activity of Au-Ag alloy NPs for in situ chemical process monitoring. 


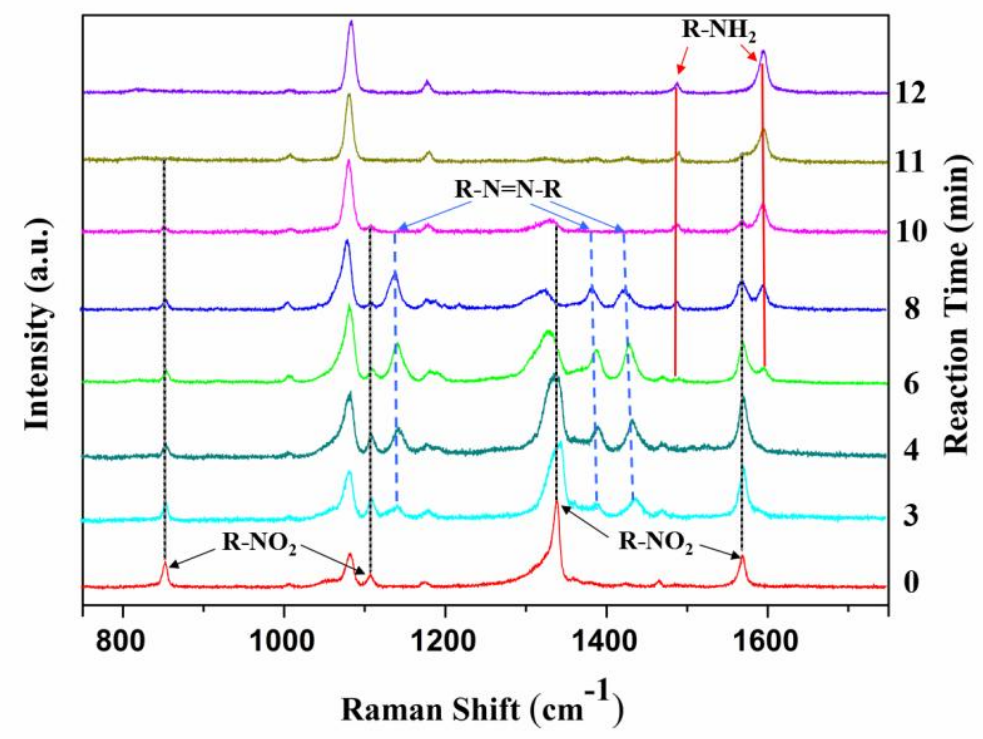

Fig. 4. SERS spectra recorded during the Au-Ag alloy NPs reduction of an aromatic nitro compound after addition of $\mathrm{NaBH}_{4}$ solution for different reaction time.

However, different results were observed from reaction system that contained Au or Ag NPs. For SAM of 4-NTP on Au NPs, the SERS spectrum with no obvious change was observed under the similar conditions with the catalytic reaction of $\mathrm{Au}-\mathrm{Ag}$ alloy NPs (Fig. S3), demonstrating that Au NPs have a very low catalytic activity in this case [37]. While for a SAM of 4-NTP on Ag NPs experiment, vibrational Raman bands from 4,4'-dimercapto-azobenzene $\left(4,4^{\prime}\right.$-DMAB) were detected, which are at 1144, 1390, and $1436 \mathrm{~cm}^{-1}$ due to $\mathrm{C}-\mathrm{N}$ symmetric stretching, $\mathrm{N}=\mathrm{N}$ stretching, and C-H in-plane bending modes (Fig. S4). Interestingly, these three bands are assigned to 4-NTP instead of 4-ATP in SERS spectrum [12, 25]. Obviously, the catalytic activity of Au-Ag alloy NPs is better than the conventional monometallic Au or Ag NPs. This may be caused by the dramatic increase of the catalytic activity when alloying of silver with gold.

It is well-known that $\mathrm{Au}$ NPs less than $5 \mathrm{~nm}$ in diameter have remarkable catalytic activity, but the plasmon resonance is weak due to small scattering cross section [38]. However, Au-Ag NPs with a size of $33 \mathrm{~nm}$ shows high catalytic activity by using SERS as an ultrasensitive spectroscopic technique to precisely monitor the process in present work. This observation suggests that the size and structure of Au-Ag alloy 
NPs seem not are a very critical factors for the reaction system. Although we are still on the way to understand the underlying mechanism of the catalysis by this alloy NPs, we believe that the presence of Ag plays a very important role in $\mathrm{Au}-\mathrm{Ag}$ alloy NPs-catalyzed reaction. Au-Ag alloy NPs have presented a strongly synergistic effect in plasmonic and catalytic activity, which could bring us a new metal nanosystem with good catalytic property.

\section{Conclusion}

In summary, a simple facile synthetic protocol was established for preparation the monodispersed Au-Ag alloy NPs, which integrate both good plasmonic property of $\mathrm{Ag}$ and outstanding stability of $\mathrm{Au}$ into a single bifunctional entity. In addition, the Au-Ag alloy NPs exhibit very well catalytic activity and strong SERS effect, which may be due to synergistic effect. The catalytic activity of Au-Ag alloy NPs and the mechanism of Au-Ag alloy NPs-catalyzed reactions are investigated with 4-NTP molecules by in situ real-time quantitative SERS, which ensure the signal detection on the intermediate and final products. The study offers a new strategy that could be applied for the preparation of other alloy catalysts for diverse uses by synergy effects. Moreover, with the development of SERS-based synergy technique and catalytic products, we believed that the unprecedented improvement in catalytic reaction of aromatic nitro compound is on the way to achieve, which very possibly opens a new window for environmental protection.

\section{Acknowledgments}

The work is supported by the National Science Foundation of China (Grant 11174190, 11574190), the Fundamental Research Funds for the Central Universities (Grants GK201504005), and Innovation Funds of Graduate Programs, SNNU. (Grants 2015CXB011).

\section{References}

[1] P. Christopher, H. L. Xin, S. Linic, Visible-Light-Enhanced Catalytic Oxidation Reactions on Plasmonic Silver Nanostructures, Nat. Chem. 3 (2011) 467-472. 
[2] P. I. Geshev, U. C. Fischer, H. Fuchs, Calculation of tip enhanced Raman scattering caused by nanoparticle plasmons acting on a molecule placed near a metallic film, Phys. Rev. B. 81 (2010) 125441.

[3] J. Homola, S. S. Yee, G. Gauglitz, Surface plasmon resonance sensors: review, Sensors and Actuators B 54 (1999) 3-15.

[4] J. W. Kim, E. Galanzha, E. V. Shashkov, H. M. Moon, V. P. Zharov, Golden Carbon Nanotubes as Multimodal Photoacoustic and Photothermal High-contrast Molecular Agents, Nat. Nanotech. 4 (2009) 688-694.

[5] J. Dong, S. X. Qu, H. R. Zheng, Z. L. Zhang, J. N. Li, Y. P. Huo, G. A. Li, Simultaneous SEF and SERRS from silver fractal-like nanostructure, Sensors and Actuators B 191 (2014) 595- 599.

[6] I. Vassalini, E. Rotunno, L. Lazzarini, I. Alessandri, “Stainless” Gold Nanorods: Preserving Shape, Optical Properties, and SERS Activity in Oxidative Environment, ACS Appl. Mater. Interfaces 7 (2015) 18794-18802.

[7] J. N. Li, T. Z. Liu, H. R. Zheng, F. Gao, J. Dong, Z. L. Zhang, Z. Y. Zhang, Plasmon resonances and strong electric field enhancements in side-by-side tangent nanospheroid homodimers, Opt. Express 21(2013) 17176-17185.

[8] X. C. Ye, J. Chen, B. T. Diroll, C. B. Murray, Tunable Plasmonic Coupling in Self-Assembled Binary Nanocrystal Superlattices Studied by Correlated Optical Microspectrophotometry and Electron Microscopy, Nano Lett. 13(2013) 1291-1297 .

[9] J. Dong, Z. L. Zhang, H. R. Zheng, M. T. Sun, Recent Progress on Plasmon-Enhanced Fluorescence, Nanophotonics 4 (2015) 472- 490.

[10]C. Ayala-Orozco, J. G. Liu, M. W. Knight, Y. M. Wang, J. Day, P. Nordlander, N. J. Halas, Fluorescence Enhancement of Molecules Inside a Gold Nanomatryoshka, Nano Lett. 14 (2014) 2926-2933.

[11]C. M. Cobley, S. E. Skrabalak, D. J. Campbell, Y. N. Xia, Shape-controlled synthesis of silver nanoparticles for plasmonic and sensing applications, Plasmonics 9 
(2009) 171-179.

[12]E. Katz, I. Willner, Integrated Nanoparticle-Biomolecule Hybrid Systems: Synthesis, Properties, and Applications, Angew. Chem. Int. Ed. 43 (2004) 6042-6108.

[13]M. Salmistraro, A. Schwartzberg, W. Bao, L. E. Depero, A. Weber-Bargioni, S. Cabrini, I. Alessandri, Triggering and Monitoring Plasmon-Enhanced Reactions by Optical Nanoantennas Coupled to Photocatalytic Beads, Small 9 (2013) 3301- 3307.

[14]B. Chowdhury, J. J. Bravo-Suárez, N. Mimura, J. Q. Lu, K. K. Bando, S. Tsubota, M. Haruta, In Situ UV-vis and EPR Study on the Formation of Hydroperoxide Species during Direct Gas Phase Propylene Epoxidation over Au/Ti-SiO2 Catalyst, J. Phys. Chem. B 110 (2006) 22995-22999.

[15]G. Richner, J. A. Bokhoven, Y. M. Neuhold, M. Makosch, K. Hungerbühler, In situ Infrared Monitoring of the Solid/Liquid Catalyst Interface during the Three-Phase Hydrogenation of Nitrobenzene over Nanosized $\mathrm{Au}$ on $\mathrm{TiO}_{2}$, Phys. Chem. Chem. Phys. 13 (2011) 12463.

[16]M. T. Sun, H. X. Xu, Novel Application of Plasmonics: Plasmon-Driven Surface-Catalyzed Reactions, small 8 (2012) 2777-2787.

[17]Z. L. Zhang, T. Deckert-Gaudig, V. Deckert, Label-free monitoring of plasmonic catalysis on the nanoscale, Analyst 140 (2015) 4325.

[18]H. Huang, H. Li, J. J. Feng, A. J. Wang, One-step green synthesis of fluorescent bimetallic $\mathrm{Au} / \mathrm{Ag}$ nanoclusters for temperature sensing and in vitro detection of $\mathrm{Fe}^{3+}$, Sensors and Actuators B 223 (2016) 550- 556.

[19]L. L. Kang, P. Xu, B. Zhang, H. H. Tsai, X. J. Han, H. L. Wang, Laser wavelength- and power-dependent plasmon-driven chemical reactions monitored using single particle surface enhanced Raman spectroscopy, Chem. Commun. 49 (2013) 3389-3478.

[20]G. Sinha, L. E. Depero, I. Alessandri, Recyclable SERS Substrates Based on Au-Coated ZnO Nanorods, ACS Appl. Mater. Interfaces 3 (2011) 2557- 2563. 
[21] J. F. Li, Y. F. Huang, Y. Ding, Z. L. Yang, S. B. Li, X. S. Zhou, F. R. Fan, W. Zhang, Z. Y. Zhou, D. Y. Wu, B. Ren, Z. L. Wang, Z. Q. Tian, Shell-Isolated Nanoparticle-Enhanced Raman Spectroscopy, Nature 464 (2010) 392-395.

[22] Y. Wei, C. Cao, R. C. Jin,C. A. Mirkin, Nanoparticles with Raman Spectroscopic Fingerprints for DNA and RNA Detection, Science 297 (2002) 1536-1540.

[23]S. M. Nie, S. R. Emory, Probing Single Molecules and Single Nanoparticles by Surface-Enhanced Raman Scattering, Science 275 (1997) 1102-1106.

[24] K. N. Heck, B. G. Janesko, G. E. Scuseria, N. J. Halas, M. S. Wong, Observing Metal-Catalyzed Chemical Reactions in Situ Using Surface-Enhanced Raman Spectroscopy on Pd-Au Nanoshells, J. Am. Chem. Soc. 130 (2008) 16592-16600.

[25]H. Jing, Q. F. Zhang, N. Large, C. M. Yu, D. A. Blom, P. Nordlander, H. Wang, Tunable Plasmonic Nanoparticles with Catalytically Active High-Index Facets, Nano Lett. 14 (2014) 3674- 3682.

[26]M. C. Daniel, D. Astruc, Gold Nanoparticles: Assembly, Supramolecular Chemistry, Quantum-Size-Related Properties, and Applications toward Biology, Catalysis, and Nanotechnology, Chem. Rev. 104 (2004) 293-346.

[27] A. S. K. Hashmi, G. J. Hutchings, Gold Catalysis, Angew. Chem. Int. Ed. 45 (2006) 7896-7936.

[28]C. B. Gao, Y. X. Hu, M. S. Wang, M. F. Chi, Y. D. Yin, Fully Alloyed Ag/Au Nanospheres: Combining the Plasmonic Property of Ag with the Stability of Au, J. Am. Chem. Soc. 136 (2014) 7474-7479.

[29]P. B. Johnson, R. W. Christy, Optical constants of the noble metals, Phys. Rev. B 6 (1972) 4370-4379.

[30]D. Kim, J. Resasco, Y. Yu, A. M. Asiri, P. D. Yang, Synergistic Geometric and Electronic Effects for Electrochemical Reduction of Carbon Dioxide using Gold-Copper Bimetallic Nanoparticles, Nat. Commun. 52 (2014) 4948-4956.

[31]A. Panáček, L. Kvítek, R. Prucek, M. Kolář, R. Večeřová, N. Pizúrová, V.K. 
Sharma, T. Nev ě čná, R. Zbo řil, Silver Colloid Nanoparticles: Synthesis, Characterization, and Their Antibacterial Activity, J. Phys. Chem. B. 110 (2006) $16248-16253$.

[32]M. Rycenga, C. M. Cobley, J. Zeng, W. Y. Li, C. H. Moran, Q. Zhang, D. Qin, Y. N. Xia, Controlling the Synthesis and Assembly of Silver Nanostructures for Plasmonic Applications, Chem Rev. 111 (2011) 3669-3712.

[33] N. G. Bastús, F. Merkoçi, J. Piella, V. Puntes, Synthesis of Highly Monodisperse Citrate-Stabilized Silver Nanoparticles of up to $200 \mathrm{~nm}$ : Kinetic Control and Catalytic Properties, Chem. Mater. 26 (2014) 2836-2846.

[34] Y. F. Huang, M. Zhang, L. B. Zhao, J. M. Feng, D. Y. Wu, B. Ren, Z. Q. Tian, Activation of Oxygen on Gold and Silver Nanoparticles Assisted by Surface Plasmon Resonances, Angew. Chem. Int. Ed. 53 (2014) 2353-2357.

[35] J. F. Huang, Y. H. Zhu, M. Lin, Q. X. Wang, L. Zhao, Y. Yang, K. X. Yao, Y. Han, Site-Specific Growth of Au-Pd Alloy Horns on Au Nanorods: A Platform for Highly Sensitive Monitoring of Catalytic Reactions by Surface Enhancement Raman Spectroscopy, J. Am. Chem. Soc. 135 (2013) 8552-8561.

[36]P. Hervés, M. Pérez-Lorenzo, L. M. Liz-Marzán, J. Dzubiella, Y. Lu, M. Ballauff, Catalysis by Metallic Nanoparticles in Aqueous Solution: Model Reactions, Chem. Soc. Rev. 41 (2012) 5577-5587.

[37]W. Xie, B. Walkenfort, S. Schlücker, Label-Free SERS Monitoring of Chemical Reactions Catalyzed by Small Gold Nanoparticles Using 3D Plasmonic Superstructures, J. Am. Chem. Soc. 135(2013) 1657-1660.

[38] J. A. Scholl, A. L. Koh, J. A. Dionne, Quantum plasmon resonances of individual metallic nanoparticles, Nature 483 (2012) 421-427. 